\title{
On Bott-Chern cohomology of compact complex surfaces
}

\author{
Daniele Angella • Georges Dloussky • \\ Adriano Tomassini
}

Received: 20 February 2014 / Accepted: 24 September 2014 / Published online: 21 October 2014

(C) Fondazione Annali di Matematica Pura ed Applicata and Springer-Verlag Berlin Heidelberg 2014

\begin{abstract}
We study Bott-Chern cohomology on compact complex non-Kähler surfaces. In particular, we compute such a cohomology for compact complex surfaces in class VII and for compact complex surfaces diffeomorphic to solvmanifolds.
\end{abstract}

Keywords Compact complex surfaces · Bott-Chern cohomology · Class VII · Solvmanifold Mathematics Subject Classification $32 \mathrm{C} 35 \cdot 57 \mathrm{~T} 15 \cdot 32 \mathrm{~J} 15$

This work was supported by the Project PRIN "Varietà reali e complesse: geometria, topologia e analisi armonica", by the Projects FIRB "Geometria Differenziale Complessa e Dinamica Olomorfa" and "Geometria Differenziale e Teoria Geometrica delle Funzioni", by GNSAGA of INdAM, and by ANR "Méthodes nouvelles en géométrie non kählerienne".

D. Angella

Istituto Nazionale di Alta Matematica, Rome, Italy

Present address:

D. Angella

Dipartimento di Matematica e Informatica, Università di Parma, Parco Area delle Scienze 53/A,

43124 Parma, Italy

e-mail: daniele.angella@gmail.com

G. Dloussky

CNRS, Centrale Marseille, I2M, UMR 7373, Aix-Marseille University, 1345339 rue F. Joliot-Curie, 13411 Marseille Cedex 13, France

e-mail: georges.dloussky@univ-amu.fr

A. Tomassini $(\bowtie)$

Dipartimento di Matematica e Informatica, Università di Parma, Parco Area delle Scienze 53/A,

43124 Parma, Italy

e-mail: adriano.tomassini@unipr.it 


\section{Introduction}

For a given complex manifold $X$, many cohomological invariants can be defined, and many are known for compact complex surfaces.

Among these, one can consider Bott-Chern and Aeppli cohomologies. They are defined as follows:

$$
H_{B C}^{\bullet, \bullet}(X):=\frac{\operatorname{ker} \partial \cap \operatorname{ker} \bar{\partial}}{\operatorname{im} \partial \bar{\partial}} \quad \text { and } \quad H_{A}^{\bullet, \bullet}(X):=\frac{\operatorname{ker} \partial \bar{\partial}}{\operatorname{im} \partial+\operatorname{im} \bar{\partial}} .
$$

Note that the identity induces natural maps

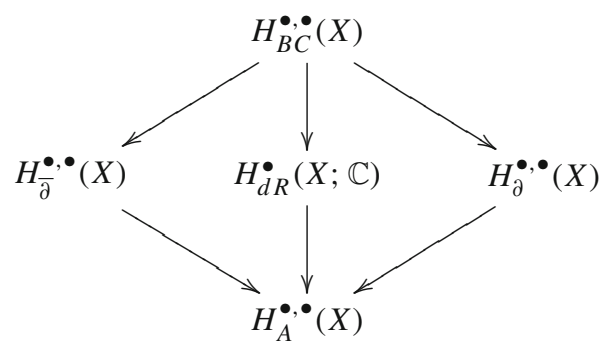

where $H_{\bar{\partial}}^{\bullet, \bullet}(X)$ denotes the Dolbeault cohomology and $H_{\partial}^{\bullet, \bullet}(X)$ its conjugate, and the maps are morphisms of (graded or bi-graded) vector spaces. For compact Kähler manifolds, the natural map $\bigoplus_{p+q=\bullet} H_{B C}^{p, q}(X) \rightarrow H_{d R}^{\bullet}(X ; \mathbb{C})$ is an isomorphism.

Assume that $X$ is compact. The Bott-Chern and Aeppli cohomologies are isomorphic to the kernel of suitable 4th-order differential elliptic operators, see [19, Section 2.b, Section 2.c]. In particular, they are finite-dimensional vector spaces. In fact, fixed a Hermitian metric $g$, its associated $\mathbb{C}$-linear Hodge-*-operator induces the isomorphism

$$
H_{B C}^{p, q}(X) \stackrel{\simeq}{\rightarrow} H_{A}^{n-q, n-p}(X),
$$

for any $p, q \in\{0, \ldots, n\}$, where $n$ denotes the complex dimension of $X$. In particular, for any $p, q \in\{0, \ldots, n\}$, one has

$$
\operatorname{dim}_{\mathbb{C}} H_{B C}^{p, q}(X)=\operatorname{dim}_{\mathbb{C}} H_{B C}^{q, p}(X)=\operatorname{dim}_{\mathbb{C}} H_{A}^{n-p, n-q}(X)=\operatorname{dim}_{\mathbb{C}} H_{A}^{n-q, n-p}(X) .
$$

For the Dolbeault cohomology, the Frölicher inequality relates the Hodge numbers and the Betti numbers: for any $k \in\{0, \ldots, 2 n\}$,

$$
\sum_{p+q=k} \operatorname{dim}_{\mathbb{C}} H_{\bar{\partial}}^{p, q}(X) \geq \operatorname{dim}_{\mathbb{C}} H_{d R}^{k}(X ; \mathbb{C}) .
$$

Similarly, for Bott-Chern cohomology, the following inequality à la Frölicher has been proven in $[3$, Theorem A]: for any $k \in\{0, \ldots, n\}$,

$$
\sum_{p+q=k}\left(\operatorname{dim}_{\mathbb{C}} H_{B C}^{p, q}(X)+\operatorname{dim}_{\mathbb{C}} H_{A}^{p, q}(X)\right) \geq 2 \operatorname{dim}_{\mathbb{C}} H_{d R}^{k}(X ; \mathbb{C}) .
$$

The equality in the Frölicher inequality characterizes the degeneration of the Frölicher spectral sequence at the first level. This always happens for compact complex surfaces. On the other side, in [3, Theorem B], it is proven that the equality in the inequality à la Frölicher for the Bott-Chern cohomology characterizes the validity of the $\partial \bar{\partial}$-Lemma, namely the property that every $\partial$-closed $\bar{\partial}$-closed d-exact form is $\partial \bar{\partial}$-exact too, [8]. The validity of the $\partial \bar{\partial}$-Lemma 
implies that the first Betti number is even, which is equivalent to Kählerness for compact complex surfaces. Therefore, the positive integer numbers

$$
\Delta^{k}:=\sum_{p+q=k}\left(\operatorname{dim}_{\mathbb{C}} H_{B C}^{p, q}(X)+\operatorname{dim}_{\mathbb{C}} H_{A}^{p, q}(X)\right)-2 b_{k} \in \mathbb{N},
$$

varying $k \in\{1,2\}$, measure the non-Kählerness of compact complex surfaces $X$.

Compact complex surfaces are divided into seven classes, according to the Kodaira and Enriques classification, see, e.g., [4]. In this note, we compute Bott-Chern cohomology for some classes of compact complex (non-Kähler) surfaces. In particular, we are interested in studying the relations between Bott-Chern cohomology and de Rham cohomology, looking at the injectivity of the natural map $H_{B C}^{2,1}(X) \rightarrow H_{d R}^{3}(X ; \mathbb{C})$. This can be intended as a weak version of the $\partial \bar{\partial}$-Lemma, compare also [10].

More precisely, we start by proving that the non-Kählerness for compact complex surfaces is encoded only in $\Delta^{2}$, namely $\Delta^{1}$ is always zero. This gives a partial answer to a question by T. C. Dinh to the third author.

Theorem 1.1 Let $X$ be a compact complex surface. Then:

(i) the natural map $H_{B C}^{2,1}(X) \rightarrow H_{\bar{\partial}}^{2,1}(X)$ induced by the identity is injective;

(ii) $\Delta^{1}=0$.

In particular, the non-Kählerness of $X$ is measured by just $\Delta^{2} \in \mathbb{N}$.

For compact complex surfaces in class VII, we show the following result, where we denote $h_{B C}^{p, q}:=\operatorname{dim}_{\mathbb{C}} H_{B C}^{p, q}(X)$ for $p, q \in\{0,1,2\}$.

Theorem 1.2 The Bott-Chern numbers of compact complex surfaces in class VII are:

$$
\begin{aligned}
& h_{B C}^{0,0}=1 \\
& h_{B C}^{2,0}=0 \begin{array}{l}
h_{B C}^{1,0}=0 \\
h_{B C}^{2,1}=1
\end{array} h_{B C}^{1,1}=b_{2}+1 \stackrel{h_{B C}^{0,1}=0}{h_{B C}^{1,2}=1} h_{B C}^{0,2}=0 \\
& h_{B C}^{2,2}=1 \text {. }
\end{aligned}
$$

According to Theorem 1.1, the natural map $H_{B C}^{2,1}(X) \rightarrow H_{\bar{\partial}}^{2,1}(X)$ is injective for any compact complex surface. One is then interested in studying the injectivity of the natural map $H_{B C}^{2,1}(X) \rightarrow H_{d R}^{3}(X ; \mathbb{C})$ induced by the identity, at least for compact complex surfaces diffeomorphic to solvmanifolds. In fact, by definition, the property of satisfying the $\partial \bar{\partial}$-Lemma [8], is equivalent to the natural map $\bigoplus_{p+q=\bullet} H_{B C}^{p, q}(X) \rightarrow H_{d R}^{\bullet}(X ; \mathbb{C})$ being injective. Note that, for a compact complex manifold of complex dimension $n$, the injectivity of the map $H_{B C}^{n, n-1}(X) \rightarrow H_{d R}^{2 n-1}(X ; \mathbb{C})$ implies the $(n-1, n)$-th weak $\partial \bar{\partial}$-Lemma in the sense of $\mathrm{Fu}$ and Yau [10, Definition 5].

We then compute the Bott-Chern cohomology for compact complex surfaces diffeomorphic to solvmanifolds, according to the list given by Hasegawa [11], see Theorem 5.1. More precisely, we prove that the cohomologies can be computed by using just leftinvariant forms. Furthermore, for such complex surfaces, we note that the natural map $H_{B C}^{2,1}(X) \rightarrow H_{d R}^{3}(X ; \mathbb{C})$ is injective, see Theorem 5.2.

We note that the above classes do not exhaust the set of compact complex non-Kähler surfaces, the cohomologies of elliptic surfaces being still unknown. 


\section{Non-Kählerness of compact complex surfaces and Bott-Chern cohomology}

We recall that, for a compact complex manifold of complex dimension $n$, for $k \in\{0, \ldots, 2 n\}$, we define the "non-Kählerness" degrees, [3, Theorem A],

$$
\Delta^{k}:=\sum_{p+q=k}\left(h_{B C}^{p, q}+h_{B C}^{n-q, n-p}\right)-2 b_{k} \in \mathbb{N},
$$

where we use the duality in [19, §2.c] giving $h_{B C}^{p, q}:=\operatorname{dim}_{\mathbb{C}} H_{B C}^{p, q}(X)=\operatorname{dim}_{\mathbb{C}} H_{A}^{n-q, n-p}(X)$. According to [3, Theorem B], $\Delta^{k}=0$ for any $k \in\{0, \ldots, 2 n\}$ if and only if $X$ satisfies the $\partial \bar{\partial}$-Lemma, namely every $\partial$-closed $\bar{\partial}$-closed d-exact form is $\partial \bar{\partial}$-exact too. In particular, for a compact complex surface $X$, the condition $\Delta^{1}=\Delta^{2}=0$ is equivalent to $X$ being Kähler, the first Betti number being even [14,17,20], see also [15, Corollaire 5.7], and [5, Theorem $11]$.

We prove that $\Delta^{1}$ is always zero for any compact complex surface. In particular, a sufficient and necessary condition for compact complex surfaces to be Kähler is $\Delta^{2}=0$.

Theorem 2.1 Let $X$ be a compact complex surface. Then:

(i) the natural map $H_{B C}^{2,1}(X) \rightarrow H_{\bar{\partial}}^{2,1}(X)$ induced by the identity is injective;

(ii) $\Delta^{1}=0$.

In particular, the non-Kählerness of $X$ is measured by just $\Delta^{2} \in \mathbb{N}$.

Proof (i) Let $\alpha \in \wedge^{2,1} X$ be such that $[\alpha]=0 \in H_{\bar{\partial}}^{2,1}(X)$. Let $\beta \in \wedge^{2,0} X$ be such that $\alpha=\bar{\partial} \beta$. Fix a Hermitian metric $g$ on $X$, and consider the Hodge decomposition of $\beta$ with respect to the Dolbeault Laplacian $\bar{\square}$ : let $\beta=\beta_{h}+\bar{\partial}^{*} \lambda$ where $\beta_{h} \in \wedge^{2,0} X \cap \operatorname{ker} \bar{\square}$, and $\lambda \in \wedge^{2,1} X$. Therefore, we have

$$
\alpha=\bar{\partial} \beta=\bar{\partial}^{*} \lambda=-\bar{\partial} * \underbrace{(\partial * \lambda)}_{\in \wedge^{2,0} X}=-\bar{\partial}(\partial * \lambda)=\partial \bar{\partial}(* \lambda),
$$

where we have used that any $(2,0)$-form is primitive and hence, by the Weil identity, is self-dual. In particular, $\alpha$ is $\partial \bar{\partial}$-exact, so it induces a zero class in $H_{B C}^{2,1}(X)$.

(ii) On the one hand, note that

$$
\begin{aligned}
H_{B C}^{1,0}(X) & =\frac{\operatorname{ker} \partial \cap \operatorname{ker} \bar{\partial} \cap \wedge^{1,0} X}{\operatorname{im} \partial \bar{\partial}}=\operatorname{ker} \partial \cap \operatorname{ker} \bar{\partial} \cap \wedge^{1,0} X \\
& \subseteq \operatorname{ker} \bar{\partial} \cap \wedge^{1,0} X=\frac{\operatorname{ker} \bar{\partial} \cap \wedge^{1,0} X}{\operatorname{im} \bar{\partial}}=H_{\bar{\partial}}^{1,0}(X) .
\end{aligned}
$$

It follows that

$$
\operatorname{dim}_{\mathbb{C}} H_{B C}^{0,1}(X)=\operatorname{dim}_{\mathbb{C}} H_{B C}^{1,0}(X) \leq \operatorname{dim}_{\mathbb{C}} H_{\bar{\partial}}^{1,0}(X)=b_{1}-\operatorname{dim}_{\mathbb{C}} H_{\bar{\partial}}^{0,1}(X),
$$

where we use that the Frölicher spectral sequence degenerates, hence in particular $b_{1}=$ $\operatorname{dim}_{\mathbb{C}} H_{\bar{\partial}}^{1,0}(X)+\operatorname{dim}_{\mathbb{C}} H_{\bar{\partial}}^{0,1}(X)$.

On the other hand, by part (i), we have

$$
\operatorname{dim}_{\mathbb{C}} H_{B C}^{1,2}(X)=\operatorname{dim}_{\mathbb{C}} H_{B C}^{2,1}(X) \leq \operatorname{dim}_{\mathbb{C}} H_{\bar{\partial}}^{2,1}(X)=\operatorname{dim}_{\mathbb{C}} H_{\bar{\partial}}^{0,1}(X),
$$

where we use the Kodaira and Serre duality $H_{\bar{\partial}}^{2,1}(X) \simeq H^{1}\left(X ; \Omega_{X}^{2}\right) \simeq H^{1}\left(X ; \mathcal{O}_{X}\right) \simeq$ $H_{\bar{\partial}}^{0,1}(X)$. 
By summing up, we get

$$
\begin{aligned}
\Delta^{1} & =\operatorname{dim}_{\mathbb{C}} H_{B C}^{0,1}(X)+\operatorname{dim}_{\mathbb{C}} H_{B C}^{1,0}(X)+\operatorname{dim}_{\mathbb{C}} H_{B C}^{1,2}(X)+\operatorname{dim}_{\mathbb{C}} H_{B C}^{2,1}(X)-2 b_{1} \\
& \leq 2\left(b_{1}-\operatorname{dim}_{\mathbb{C}} H_{\bar{\partial}}^{0,1}(X)+\operatorname{dim}_{\mathbb{C}} H_{\bar{\partial}}^{0,1}(X)-b_{1}\right)=0,
\end{aligned}
$$

concluding the proof.

\section{Class VII surfaces}

In this section, we compute Bott-Chern cohomology for compact complex surfaces in class VII.

Let $X$ be a compact complex surface. By Theorem 1.1, the natural map $H_{B C}^{2,1}(X) \rightarrow$ $H_{\bar{\partial}}^{2,1}(X)$ is always injective. Consider now the case when $X$ is in class VII. If $X$ is minimal, we prove that the same holds for cohomology with values in a line bundle. We will also prove that the natural map $H_{B C}^{1,2}(X) \rightarrow H_{\bar{\partial}}^{1,2}(X)$ is not injective.

Proposition 3.1 Let $X$ be a compact complex surface in class $V I I_{0}$. Let $L \in H^{1}\left(X ; \mathbb{C}^{*}\right)=$ $\operatorname{Pic}^{0}(X)$. The natural map $H_{B C}^{2,1}(X ; L) \rightarrow H_{\bar{\partial}}^{2,1}(X ; L)$ induced by the identity is injective.

Proof Let $\alpha \in \wedge^{2,1} X \otimes L$ be a $\bar{\partial}_{L}$-exact $(2,1)$-form. We need to prove that $\alpha$ is $\partial_{L} \bar{\partial}_{L}$-exact too. Consider $\alpha=\bar{\partial}_{L} \vartheta$, where $\vartheta \in \wedge^{2,0} X \otimes L$. In particular, $\partial_{L} \vartheta=0$, hence $\bar{\vartheta}$ defines a class in $H_{\bar{\partial}}^{0,2}(X ; L)$. Note that $H_{\bar{\partial}}^{0,2}(X ; L) \simeq H^{2}\left(X ; \mathcal{O}_{X}(L)\right) \simeq H^{0}\left(X ; K_{X} \otimes L^{-1}\right)=\{0\}$ for surfaces of class $\mathrm{VII}_{0}$, [9, Remark 2.21]. It follows that $\bar{\vartheta}=-\bar{\partial}_{L} \bar{\eta}$ for some $\eta \in \wedge^{1,0} X \otimes L$. Hence $\alpha=\partial_{L} \bar{\partial}_{L} \eta$, that is, $\alpha$ is $\partial_{L} \bar{\partial}_{L}$-exact.

We now compute the Bott-Chern cohomology of class VII surfaces.

Theorem 3.2 The Bott-Chern numbers of compact complex surfaces in class VII are:

$$
\begin{aligned}
& h_{B C}^{0,0}=1 \\
& h_{B C}^{2,0}=0 \begin{array}{l}
h_{B C}^{1,0}=0 \\
h_{B C}^{2,1}=1 \quad \begin{array}{c}
h_{B C}^{1,1}=b_{2}+1 \\
h_{B C}^{0,1}=1 .
\end{array} \quad h_{B C}^{1,2}=1
\end{array}
\end{aligned}
$$

Proof It holds $H_{B C}^{1,0}(X)=\frac{\operatorname{ker} \partial \cap \operatorname{ker} \bar{\partial} \cap \wedge^{1,0} X}{\operatorname{im} \partial \bar{\partial}}=\operatorname{ker} \partial \cap \operatorname{ker} \bar{\partial} \cap \wedge^{1,0} X \subseteq \operatorname{ker} \bar{\partial} \cap \wedge^{1,0} X=$ $\frac{\operatorname{ker} \bar{\partial} \cap \wedge^{1,0} X}{\operatorname{im} \bar{\partial}}=H_{\bar{\partial}}^{1,0}(X)=\{0\}$ hence $h_{B C}^{1,0}=h_{B C}^{0,1}=0$.

On the other side, by Theorem 1.1, $0=\Delta^{1}=2\left(h_{B C}^{1,0}+h_{B C}^{2,1}-b_{1}\right)=2\left(h_{B C}^{2,1}-1\right)$ hence $h_{B C}^{2,1}=h_{B C}^{1,2}=1$.

Similarly, it holds $H_{B C}^{2,0}(X)=\frac{\operatorname{ker} \partial \cap \operatorname{ker} \bar{\partial} \cap \wedge^{2,0} X}{\operatorname{im} \partial \bar{\partial}}=\operatorname{ker} \partial \cap \operatorname{ker} \bar{\partial} \cap \wedge^{2,0} X \subseteq \operatorname{ker} \bar{\partial} \cap \wedge^{2,0} X=$ $\frac{\operatorname{ker} \bar{\partial} \cap \wedge^{2,0} X}{\operatorname{im} \bar{\partial}}=H_{\bar{\partial}}^{2,0}(X)=\{0\}$ hence $h_{B C}^{2,0}=h_{B C}^{0,2}=0$.

Note that, from [3, Theorem A], we have $0 \leq \Delta^{2}=2\left(h_{B C}^{2,0}+h_{B C}^{1,1}+h_{B C}^{0,2}-b_{2}\right)=$ $2\left(h_{B C}^{1,1}-b_{2}\right)$ hence $h_{B C}^{1,1} \geq b_{2}$. More precisely, from [3, Theorem B] and Theorem 1.1, we have that $h_{B C}^{1,1}=b_{2}$ if and only if $\Delta^{2}=0$ if and only if $X$ satisfies the $\partial \bar{\partial}$-Lemma, in fact $X$ is Kähler, which is not the case. 
Finally, we prove that $h_{B C}^{1,1}=b_{2}+1$. Consider the following exact sequences from [21, Lemma 2.3]. More precisely, the sequence

$$
0 \rightarrow \frac{\operatorname{im~} \cap \wedge^{1,1} X}{\operatorname{im} \partial \bar{\partial}} \rightarrow H_{B C}^{1,1}(X) \rightarrow \operatorname{im}\left(H_{B C}^{1,1}(X) \rightarrow H_{d R}^{2}(X ; \mathbb{C})\right) \rightarrow 0
$$

is clearly exact. Furthermore, fix a Gauduchon metric $g$. Denote by $\omega:=g(J \cdot, \cdot \cdot)$ the $(1,1)$ form associated to $g$, where $J$ denotes the integrable almost-complex structure. By definition of $g$ being Gauduchon, we have $\partial \bar{\partial} \omega=0$. The sequence

$$
0 \rightarrow \frac{\operatorname{imd} \cap \wedge^{1,1} X}{\operatorname{im} \partial \bar{\partial}} \stackrel{\langle\cdot \mid \omega\rangle}{\rightarrow} \mathbb{C}
$$

is exact. Indeed, firstly note that for $\eta=\partial \bar{\partial} f \in \operatorname{im} \partial \bar{\partial} \cap \wedge^{1,1} X$, we have

$$
\langle\eta \mid \omega\rangle=\int_{X} \partial \bar{\partial} f \wedge \overline{* \omega}=\int_{X} \partial \bar{\partial} f \wedge \omega=\int_{X} f \partial \bar{\partial} \omega=0
$$

by applying twice the Stokes theorem. Then, we recall the argument in [21, Lemma 2.3(ii)] for proving that the map

$$
\langle\cdot \mid \omega\rangle: \frac{\operatorname{im} \mathrm{d} \cap \wedge^{1,1} X}{\operatorname{im} \partial \bar{\partial}} \rightarrow \mathbb{C}
$$

is injective. Take $\alpha=\mathrm{d} \beta \in \operatorname{im~} \mathrm{d} \cap \wedge^{1,1} X \cap \operatorname{ker}\langle\cdot \mid \omega\rangle$. Then,

$$
\langle\Lambda \alpha \mid 1\rangle=\langle\alpha \mid \omega\rangle=0,
$$

where $\Lambda$ is the adjoint operator of $\omega \wedge \cdot$ with respect to $\langle\cdot \mid \cdot \cdot\rangle$. Then, $\Lambda \alpha \in \operatorname{ker}\langle\cdot \mid 1\rangle=\operatorname{im} \Lambda \partial \bar{\partial}$, by extending [16, Corollary 7.2.9] by $\mathbb{C}$-linearity. Take $u \in \mathcal{C}^{\infty}(X ; \mathbb{C})$ such that $\Lambda \alpha=\Lambda \partial \bar{\partial} u$. Then, by defining $\alpha^{\prime}:=\alpha-\partial \bar{\partial} u$, we have $\left[\alpha^{\prime}\right]=[\alpha] \in \frac{\operatorname{im} \operatorname{d} \cap \wedge^{1,1} X}{\operatorname{im} \partial \bar{\partial}}$, and $\Lambda \alpha^{\prime}=0$, and $\alpha^{\prime}=\mathrm{d} \beta^{\prime}$ where $\beta^{\prime}:=\beta-\bar{\partial} u$. In particular, $\alpha^{\prime}$ is primitive. Since $\alpha^{\prime}$ is primitive and of type $(1,1)$, then, it is anti-self-dual by the Weil identity. Then,

$$
\begin{aligned}
\left\|\alpha^{\prime}\right\|^{2} & =\left\langle\alpha^{\prime} \mid \alpha^{\prime}\right\rangle=\int_{X} \alpha^{\prime} \wedge \overline{* \alpha^{\prime}}=-\int_{X} \alpha^{\prime} \wedge \overline{\alpha^{\prime}}=-\int_{X} \mathrm{~d} \beta^{\prime} \wedge \mathrm{d} \overline{\beta^{\prime}} \\
& =-\int_{X} \mathrm{~d}\left(\beta^{\prime} \wedge \mathrm{d} \overline{\beta^{\prime}}\right)=0
\end{aligned}
$$

and hence $\alpha^{\prime}=0$, and therefore $[\alpha]=0$.

Since the space $\frac{\operatorname{im} \mathrm{d} \cap \wedge^{1,1} X}{\operatorname{im} \partial \bar{\partial}}$ is finite-dimensional, being a subspace of $H_{B C}^{1,1}(X)$, and since the space $\operatorname{im}\left(H_{B C}^{1,1}(X) \rightarrow H_{d R}^{2}(X ; \mathbb{C})\right)$ is finite-dimensional, being a subspace of $H_{d R}^{2}(X ; \mathbb{C})$, we get that

$$
\operatorname{dim}_{\mathbb{C}} \frac{\operatorname{im~} \cap \cap \wedge^{1,1} X}{\operatorname{im} \partial \bar{\partial}} \leq \operatorname{dim}_{\mathbb{C}} \mathbb{C}=1
$$

and hence

$$
\begin{aligned}
b_{2}<\operatorname{dim}_{\mathbb{C}} H_{B C}^{1,1}(X)= & \operatorname{dim}_{\mathbb{C}} \operatorname{im}\left(H_{B C}^{1,1}(X) \rightarrow H_{d R}^{2}(X ; \mathbb{C})\right) \\
& +\operatorname{dim}_{\mathbb{C}} \frac{\operatorname{im~} \cap \wedge^{1,1} X}{\operatorname{im} \partial \bar{\partial}} \leq b_{2}+1 .
\end{aligned}
$$

We get that $\operatorname{dim}_{\mathbb{C}} H_{B C}^{1,1}(X)=b_{2}+1$. 
Finally, we prove that the natural map $H_{B C}^{1,2}(X) \rightarrow H_{\bar{\partial}}^{1,2}(X)$ is not injective.

Proposition 3.3 Let $X$ be a compact complex surface in class VII. Then, the natural map $H_{B C}^{1,2}(X) \rightarrow H_{\bar{\partial}}^{1,2}(X)$ induced by the identity is the zero map and not an isomorphism.

Proof Note that, for class VII surfaces, the pluri-genera are zero. In particular, $H_{\bar{\partial}}^{1,2}(X) \simeq$ $H_{\bar{\partial}}^{1,0}(X)=\{0\}$, by Kodaira and Serre duality. By Theorem 3.2, one has $H_{B C}^{1,2}(X) \neq\{0\}$.

\subsection{Cohomologies of Calabi-Eckmann surface}

In this section, as an explicit example, we list the representatives of the cohomologies of a compact complex surface in class VII: namely we consider the Calabi-Eckmann structure on the differentiable manifolds underlying the Hopf surfaces.

Consider the differentiable manifold $X:=\mathbb{S}^{1} \times \mathbb{S}^{3}$. As a Lie group, $\mathbb{S}^{3}=S U(2)$ has a global left-invariant co-frame $\left\{e^{1}, e^{2}, e^{3}\right\}$ such that $\mathrm{d} e^{1}=-2 e^{2} \wedge e^{3}$ and $\mathrm{d} e^{2}=2 e^{1} \wedge e^{3}$ and $\mathrm{d} e^{3}=-2 e^{1} \wedge e^{2}$. Hence, we consider a global left-invariant co-frame $\left\{f, e^{1}, e^{2}, e^{3}\right\}$ on $X$ with structure equations

$$
\left\{\begin{array}{rl}
\mathrm{d} f & =0 \\
\mathrm{~d} e^{1} & =-2 e^{2} \wedge e^{3} \\
\mathrm{~d} e^{2} & =2 e^{1} \wedge e^{3} \\
\mathrm{~d} e^{3} & =-2 e^{1} \wedge e^{2}
\end{array} .\right.
$$

Consider the left-invariant almost-complex structure defined by the $(1,0)$-forms

$$
\left\{\begin{array}{l}
\varphi^{1}:=e^{1}+i e^{2} \\
\varphi^{2}:=e^{3}+i f
\end{array}\right.
$$

By computing the complex structure equations, we get

$$
\left\{\begin{array} { l } 
{ \partial \varphi ^ { 1 } = i \varphi ^ { 1 } \wedge \varphi ^ { 2 } } \\
{ \partial \varphi ^ { 2 } = 0 }
\end{array} \quad \text { and } \quad \left\{\begin{array}{l}
\bar{\partial} \varphi^{1}=i \varphi^{1} \wedge \bar{\varphi}^{2} \\
\bar{\partial} \varphi^{2}=-i \varphi^{1} \wedge \bar{\varphi}^{1}
\end{array}\right.\right.
$$

We note that the almost-complex structure is in fact integrable.

The manifold $X$ is a compact complex manifold not admitting Kähler metrics. It is biholomorphic to the complex manifold $M_{0,1}$ considered by Calabi and Eckmann [6], see [18, Theorem 4.1].

Consider the Hermitian metric $g$ whose associated $(1,1)$-form is

$$
\omega:=\frac{i}{2} \sum_{j=1}^{2} \varphi^{j} \wedge \bar{\varphi}^{j} .
$$

As for the de Rham cohomology, from the Künneth formula we get

$$
H_{d R}^{\bullet}(X ; \mathbb{C})=\mathbb{C}\langle 1\rangle \oplus \mathbb{C}\left\langle\varphi^{2}-\bar{\varphi}^{2}\right\rangle \oplus \mathbb{C}\left\langle\varphi^{12 \overline{1}}-\varphi^{1 \overline{1} \overline{2}}\right\rangle \oplus \mathbb{C}\left\langle\varphi^{12 \overline{1} \overline{2}}\right\rangle,
$$

(where, here and hereafter, we shorten, e.g., $\varphi^{12 \overline{1}}:=\varphi^{1} \wedge \varphi^{2} \wedge \bar{\varphi}^{1}$ ).

By [12, Appendix II, Theorem 9.5], one has that a model for the Dolbeault cohomology is given by

$$
H_{\bar{\partial}}^{\bullet \bullet \bullet}(X) \simeq \bigwedge\left\langle x_{2,1}, x_{0,1}\right\rangle
$$


where $x_{i, j}$ is an element of bi-degree $(i, j)$. In particular, we recover that the Hodge numbers $\left\{h_{\bar{\partial}}^{p, q}:=\operatorname{dim}_{\mathbb{C}} H_{\bar{\partial}}^{p, q}(X)\right\}_{p, q \in\{0,1,2\}}$ are

$$
\begin{aligned}
& h_{\bar{\partial}}^{0,0}=1 \\
& h_{\bar{\partial}}^{1,0}=0 \quad h_{\bar{\partial}}^{0,1}=1 \\
& h_{\bar{\partial}}^{2,0}=0 \quad h_{\bar{\partial}}^{1,1}=0 \quad h_{\bar{\partial}}^{0,2}=0 .
\end{aligned}
$$

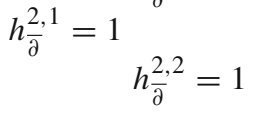

We note that the sub-complex

$$
\iota: \bigwedge\left\langle\varphi^{1}, \varphi^{2}, \bar{\varphi}^{1}, \bar{\varphi}^{2}\right\rangle \hookrightarrow \wedge^{\bullet \bullet} X
$$

is such that $H_{\bar{\partial}}(\iota)$ is an isomorphism. More precisely, we get

$$
H_{\bar{\partial}}^{\bullet, \bullet}(X)=\mathbb{C}\langle 1\rangle \oplus \mathbb{C}\left\langle\left[\varphi^{\overline{2}}\right]\right\rangle \oplus \mathbb{C}\left\langle\left[\varphi^{12 \overline{1}}\right]\right\rangle \oplus \mathbb{C}\left\langle\left[\varphi^{12 \overline{1} \overline{2}}\right]\right\rangle,
$$

where we have listed the harmonic representatives with respect to the Dolbeault Laplacian of $g$.

By [2, Theorem 1.3, Proposition 2.2], we have also $H_{B C}(\iota)$ isomorphism. In particular, we get

$$
H_{B C}^{\bullet, \bullet}(X)=\mathbb{C}\langle 1\rangle \oplus \mathbb{C}\left\langle\left[\varphi^{1 \overline{1}}\right]\right\rangle \oplus \mathbb{C}\left\langle\left[\varphi^{12 \overline{1}}\right]\right\rangle \oplus \mathbb{C}\left\langle\left[\varphi^{1 \overline{1} \overline{2}}\right]\right\rangle \oplus \mathbb{C}\left\langle\left[\varphi^{12 \overline{1} \overline{2}}\right]\right\rangle,
$$

where we have listed the harmonic representatives with respect to the Bott-Chern Laplacian of $g$.

By [19, Section 2.c], we have

$$
H_{A}^{\bullet, \bullet}(X)=\mathbb{C}\langle 1\rangle \oplus \mathbb{C}\left\langle\left[\varphi^{2}\right]\right\rangle \oplus \mathbb{C}\left\langle\left[\varphi^{\overline{2}}\right]\right\rangle \oplus \mathbb{C}\left\langle\left[\varphi^{2 \overline{2}}\right]\right\rangle \oplus \mathbb{C}\left\langle\left[\varphi^{12 \overline{1} \overline{2}}\right]\right\rangle,
$$

where we have listed the harmonic representatives with respect to the Aeppli Laplacian of $g$.

Note in particular that the natural map $H_{B C}^{2,1}(X) \rightarrow H_{\bar{\partial}}^{2,1}(X)$ induced by the identity is an isomorphism, and that the natural map $H_{B C}^{2,1}(X) \rightarrow H_{d R}^{3}(X ; \mathbb{C})$ induced by the identity is injective.

\section{Complex surfaces diffeomorphic to solvmanifolds}

Let $X$ be a compact complex surface diffeomorphic to a solvmanifold $\Gamma \backslash G$. By [11, Theorem 1], $X$ is $(A)$ either a complex torus, $(B)$ or a hyperelliptic surface, $(C)$ or a Inoue surface of type $\mathcal{S}_{M},(D)$ or a primary Kodaira surface, $(E)$ or a secondary Kodaira surface, $(F)$ or a Inoue surface of type $\mathcal{S}^{ \pm}$, and, as such, it is endowed with a left-invariant complex structure.

In each case, we recall the structure equations of the group $G$, see [11]. More precisely, take a basis $\left\{e_{1}, e_{2}, e_{3}, e_{4}\right\}$ of the Lie algebra $\mathfrak{g}$ naturally associated to $G$. We have the following commutation relations, according to [11]:

(A) differentiable structure underlying a complex torus:

$$
\left[e_{j}, e_{k}\right]=0 \quad \text { for any } \quad j, k \in\{1,2,3,4\} ;
$$

(hereafter, we write only the non-trivial commutators); 
(B) differentiable structure underlying a hyperelliptic surface:

$$
\left[e_{1}, e_{4}\right]=e_{2}, \quad\left[e_{2}, e_{4}\right]=-e_{1} ;
$$

(C) differentiable structure underlying a Inoue surface of type $\mathcal{S}_{M}$ :

$$
\left[e_{1}, e_{4}\right]=-\alpha e_{1}+\beta e_{2}, \quad\left[e_{2}, e_{4}\right]=-\beta e_{1}-\alpha e_{2}, \quad\left[e_{3}, e_{4}\right]=2 \alpha e_{3},
$$

where $\alpha \in \mathbb{R} \backslash\{0\}$ and $\beta \in \mathbb{R}$;

(D) differentiable structure underlying a primary Kodaira surface:

$$
\left[e_{1}, e_{2}\right]=-e_{3}
$$

(E) differentiable structure underlying a secondary Kodaira surface:

$$
\left[e_{1}, e_{2}\right]=-e_{3}, \quad\left[e_{1}, e_{4}\right]=e_{2}, \quad\left[e_{2}, e_{4}\right]=-e_{1} ;
$$

(F) differentiable structure underlying a Inoue surface of type $\mathcal{S}^{ \pm}$:

$$
\left[e_{2}, e_{3}\right]=-e_{1}, \quad\left[e_{2}, e_{4}\right]=-e_{2}, \quad\left[e_{3}, e_{4}\right]=e_{3} .
$$

Denote by $\left\{e^{1}, e^{2}, e^{3}, e^{4}\right\}$ the dual basis of $\left\{e_{1}, e_{2}, e_{3}, e_{4}\right\}$. We recall that, for any $\alpha \in \mathfrak{g}^{*}$, for any $x, y \in \mathfrak{g}$, it holds $\mathrm{d} \alpha(x, y)=-\alpha([x, y])$. Hence we get the following structure equations:

(A) differentiable structure underlying a complex torus:

$$
\left\{\begin{array}{l}
\mathrm{d} e^{1}=0 \\
\mathrm{~d} e^{2}=0 \\
\mathrm{~d} e^{3}=0 \\
\mathrm{~d} e^{4}=0
\end{array} ;\right.
$$

(B) differentiable structure underlying a hyperelliptic surface:

$$
\left\{\begin{array}{l}
\mathrm{d} e^{1}=e^{2} \wedge e^{4} \\
\mathrm{~d} e^{2}=-e^{1} \wedge e^{4} \\
\mathrm{~d} e^{3}=0 \\
\mathrm{~d} e^{4}=0
\end{array} ;\right.
$$

(C) differentiable structure underlying a Inoue surface of type $\mathcal{S}_{M}$ :

$$
\left\{\begin{array}{l}
\mathrm{d} e^{1}=\alpha e^{1} \wedge e^{4}+\beta e^{2} \wedge e^{4} \\
\mathrm{~d} e^{2}=-\beta e^{1} \wedge e^{4}+\alpha e^{2} \wedge e^{4} \\
\mathrm{~d} e^{3}=-2 \alpha e^{3} \wedge e^{4} \\
\mathrm{~d} e^{4}=0
\end{array}\right.
$$

(D) differentiable structure underlying a primary Kodaira surface:

$$
\left\{\begin{array}{l}
\mathrm{d} e^{1}=0 \\
\mathrm{~d} e^{2}=0 \\
\mathrm{~d} e^{3}=e^{1} \wedge e^{2} \\
\mathrm{~d} e^{4}=0
\end{array} ;\right.
$$


(E) differentiable structure underlying a secondary Kodaira surface:

$$
\left\{\begin{array}{l}
\mathrm{d} e^{1}=e^{2} \wedge e^{4} \\
\mathrm{~d} e^{2}=-e^{1} \wedge e^{4} \\
\mathrm{~d} e^{3}=e^{1} \wedge e^{2} \\
\mathrm{~d} e^{4}=0
\end{array} ;\right.
$$

(F) differentiable structure underlying a Inoue surface of type $\mathcal{S}^{ \pm}$:

$$
\left\{\begin{array}{l}
\mathrm{d} e^{1}=e^{2} \wedge e^{3} \\
\mathrm{~d} e^{2}=e^{2} \wedge e^{4} \\
\mathrm{~d} e^{3}=-e^{3} \wedge e^{4} \\
\mathrm{~d} e^{4}=0
\end{array} .\right.
$$

In cases (A), (B), (C), (D), (E), consider the $G$-left-invariant almost-complex structure $J$ on $X$ defined by

$$
J e_{1}:=e_{2} \quad \text { and } J e_{2}:=-e_{1} \quad \text { and } J e_{3}:=e_{4} \quad \text { and } J e_{4}:=-e_{3} .
$$

Consider the $G$-left-invariant $(1,0)$-forms

$$
\left\{\begin{array}{l}
\varphi^{1}:=e^{1}+i e^{2} \\
\varphi^{2}:=e^{3}+i e^{4}
\end{array} .\right.
$$

In case (F), consider the $G$-left-invariant almost-complex structure $J$ on $X$ defined by $J e_{1}:=e_{2}$ and $J e_{2}:=-e_{1}$ and $J e_{3}:=e_{4}-q e_{2}$ and $J e_{4}:=-e_{3}-q e_{1}$, where $q \in \mathbb{R}$. Consider the $G$-left-invariant $(1,0)$-forms

$$
\left\{\begin{array}{l}
\varphi^{1}:=e^{1}+i e^{2}+i q e^{4} \\
\varphi^{2}:=e^{3}+i e^{4}
\end{array} .\right.
$$

With respect to the $G$-left-invariant coframe $\left\{\varphi^{1}, \varphi^{2}\right\}$ for the holomorphic tangent bundle $T^{1,0} \Gamma \backslash G$, we have the following structure equations. (As for notation, we shorten, e.g., $\varphi^{1 \overline{2}}:=\varphi^{1} \wedge \bar{\varphi}^{2}$.)

(A) torus:

$$
\left\{\begin{array}{l}
\mathrm{d} \varphi^{1}=0 \\
\mathrm{~d} \varphi^{2}=0
\end{array}\right.
$$

(B) hyperelliptic surface:

$$
\left\{\begin{array}{l}
\mathrm{d} \varphi^{1}=-\frac{1}{2} \varphi^{12}+\frac{1}{2} \varphi^{1 \overline{2}} \\
\mathrm{~d} \varphi^{2}=0
\end{array}\right.
$$

(C) Inoue surface $\mathcal{S}_{M}$ :

$$
\left\{\begin{array}{l}
\mathrm{d} \varphi^{1}=\frac{\alpha-i \beta}{2 i} \varphi^{12}-\frac{\alpha-i \beta}{2 i} \varphi^{1 \overline{2}} \\
\mathrm{~d} \varphi^{2}=-i \alpha \varphi^{2 \overline{2}}
\end{array}\right.
$$

(where $\alpha \in \mathbb{R} \backslash\{0\}$ and $\beta \in \mathbb{R}$ ); 
(D) primary Kodaira surface:

$$
\left\{\begin{array}{l}
\mathrm{d} \varphi^{1}=0 \\
\mathrm{~d} \varphi^{2}=\frac{i}{2} \varphi^{1 \overline{1}}
\end{array}\right.
$$

(E) secondary Kodaira surface:

$$
\left\{\begin{array}{l}
\mathrm{d} \varphi^{1}=-\frac{1}{2} \varphi^{12}+\frac{1}{2} \varphi^{1 \overline{2}} \\
\mathrm{~d} \varphi^{2}=\frac{i}{2} \varphi^{1 \overline{1}}
\end{array}\right.
$$

(F) Inoue surface $\mathcal{S}^{ \pm}$:

$$
\left\{\begin{array}{l}
\mathrm{d} \varphi^{1}=\frac{1}{2 i} \varphi^{12}+\frac{1}{2 i} \varphi^{2 \overline{1}}+\frac{q i}{2} \varphi^{2 \overline{2}} \\
\mathrm{~d} \varphi^{2}=\frac{1}{2 i} \varphi^{2 \overline{2}}
\end{array} .\right.
$$

\section{Cohomologies of complex surfaces diffeomorphic to solvmanifolds}

In this section, we compute the Dolbeault and Bott-Chern cohomologies of the compact complex surfaces diffeomorphic to a solvmanifold.

We prove the following theorem.

Theorem 5.1 Let $X$ be a compact complex surface diffeomorphic to a solvmanifold $\Gamma \backslash G$; denote the Lie algebra of $G$ by $\mathfrak{g}$. Then, the inclusion $\left(\wedge^{\bullet} \cdot \mathfrak{g} \mathfrak{g}^{*}, \partial, \bar{\partial}\right) \hookrightarrow\left(\wedge^{\bullet \bullet} X, \partial, \bar{\partial}\right)$ induces an isomorphism both in Dolbeault and in Bott-Chern cohomologies. In particular, the dimensions of the de Rham, Dolbeault, and Bott-Chern cohomologies and the degrees of non-Kählerness are summarized in Table 5.

Proof Firstly, we compute the cohomologies of the sub-complex of $G$-left-invariant forms. The computations are straightforward from the structure equations.

In Tables 1, 2, 3 and 4, we summarize the results of the computations. The sub-complexes of left-invariant forms are depicted in Fig. 1 (each dot represents a generator, vertical arrows depict the $\bar{\partial}$-operator, horizontal arrows depict the $\partial$-operator, and trivial arrows are not shown.) The dimensions are listed in Table 5.

On the one side, recall that the inclusion of left-invariant forms into the space of forms induces an injective map in Dolbeault and Bott-Chern cohomologies, see, e.g., [7, Lemma 9], [1, Lemma 3.6]. On the other side, recall that the Frölicher spectral sequence of a compact complex surface $X$ degenerates at the first level, equivalently, the equalities

$$
\operatorname{dim}_{\mathbb{C}} H_{\bar{\partial}}^{1,0}(X)+\operatorname{dim}_{\mathbb{C}} H_{\bar{\partial}}^{0,1}(X)=\operatorname{dim}_{\mathbb{C}} H_{d R}^{1}(X ; \mathbb{C})
$$

and

$$
\operatorname{dim}_{\mathbb{C}} H_{\bar{\partial}}^{2,0}(X)+\operatorname{dim}_{\mathbb{C}} H_{\bar{\partial}}^{1,1}(X)+\operatorname{dim}_{\mathbb{C}} H_{\bar{\partial}}^{0,2}(X)=\operatorname{dim}_{\mathbb{C}} H_{d R}^{2}(X ; \mathbb{C})
$$

hold. By comparing the dimensions in Table 5 with the Betti numbers case by case, we find that the left-invariant forms suffice in computing the Dolbeault cohomology for each case. Then, by [1, Theorem 3.7], see also [2, Theorem 1.3, Theorem 1.6], it follows that also the Bott-Chern cohomology is computed using just left-invariant forms.

We prove the following result. 


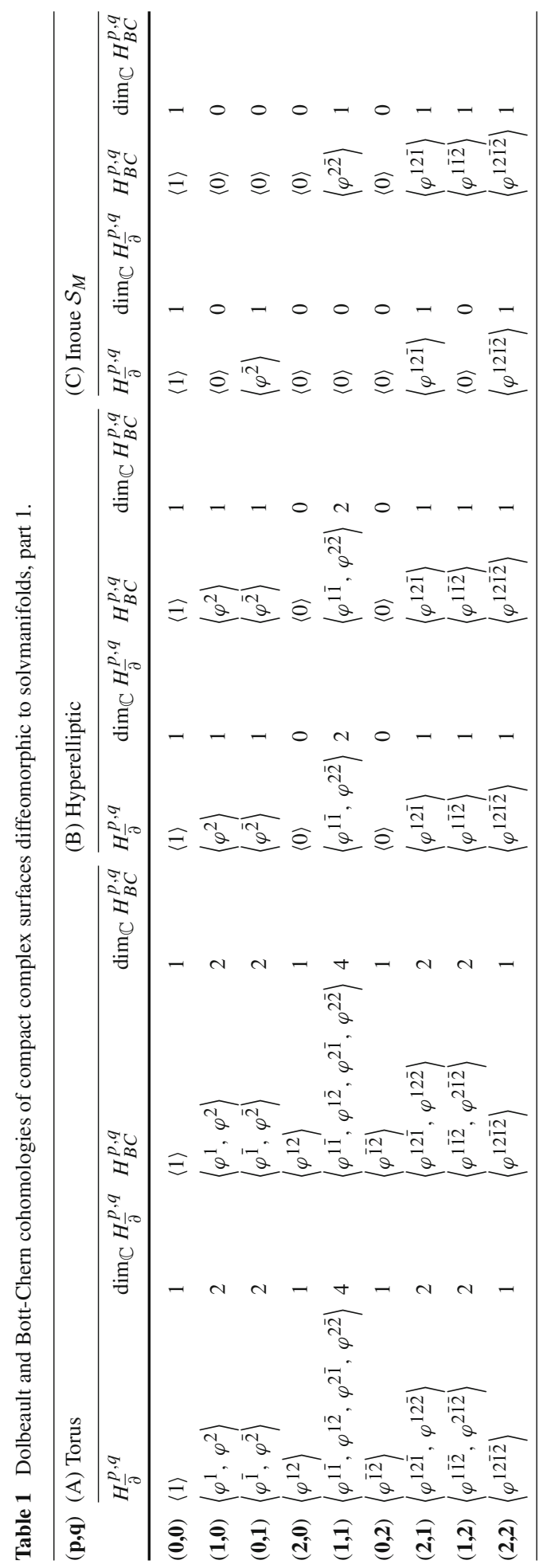




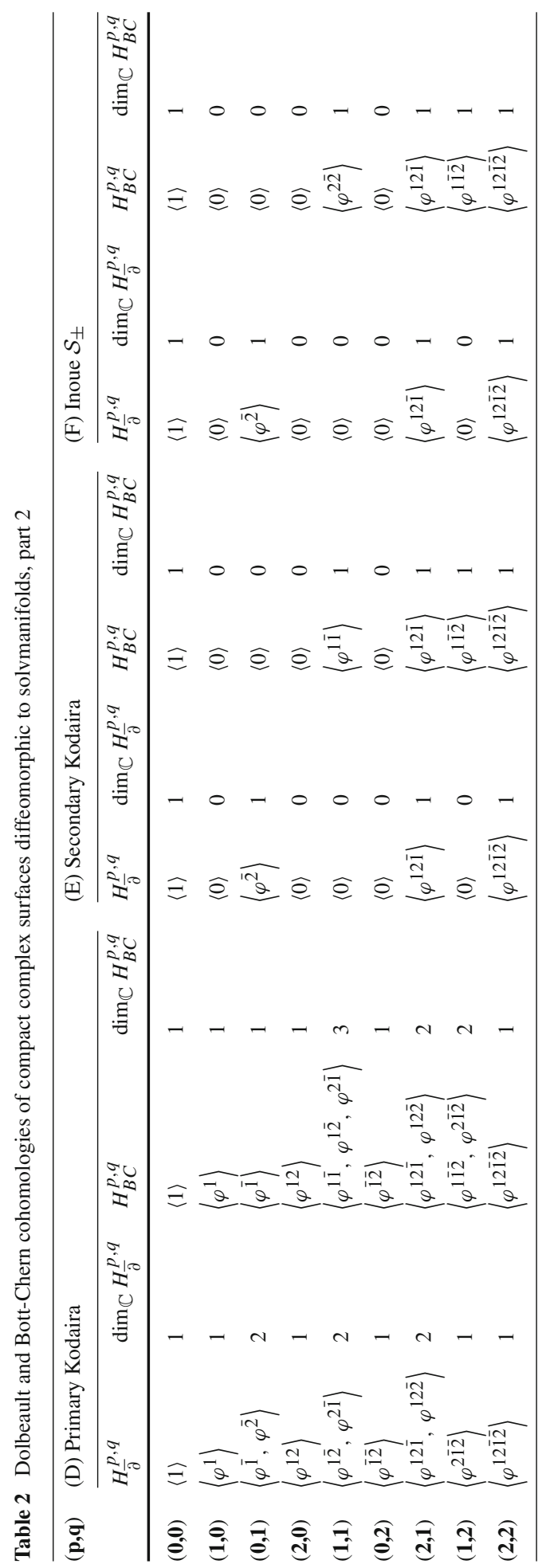




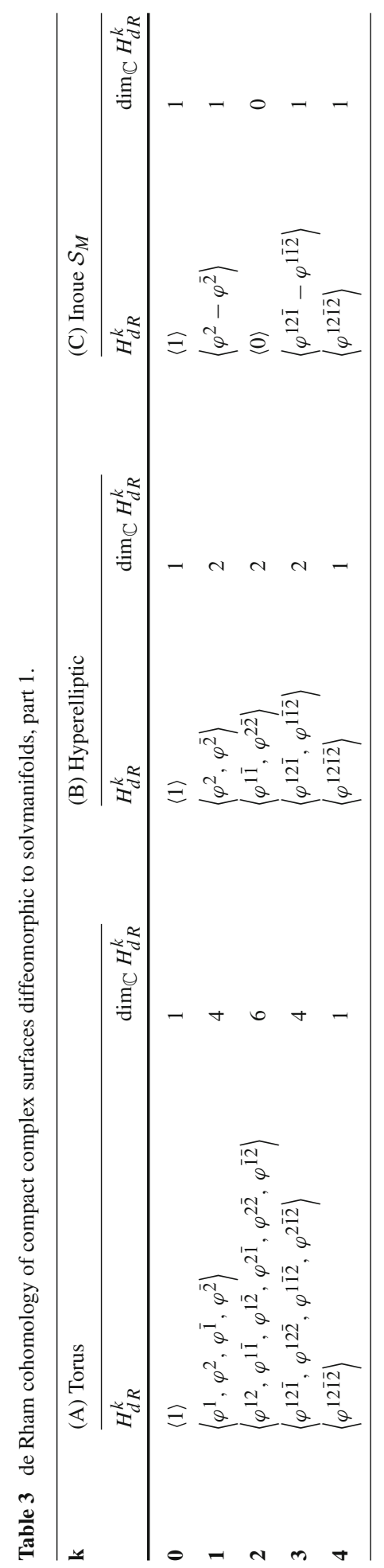




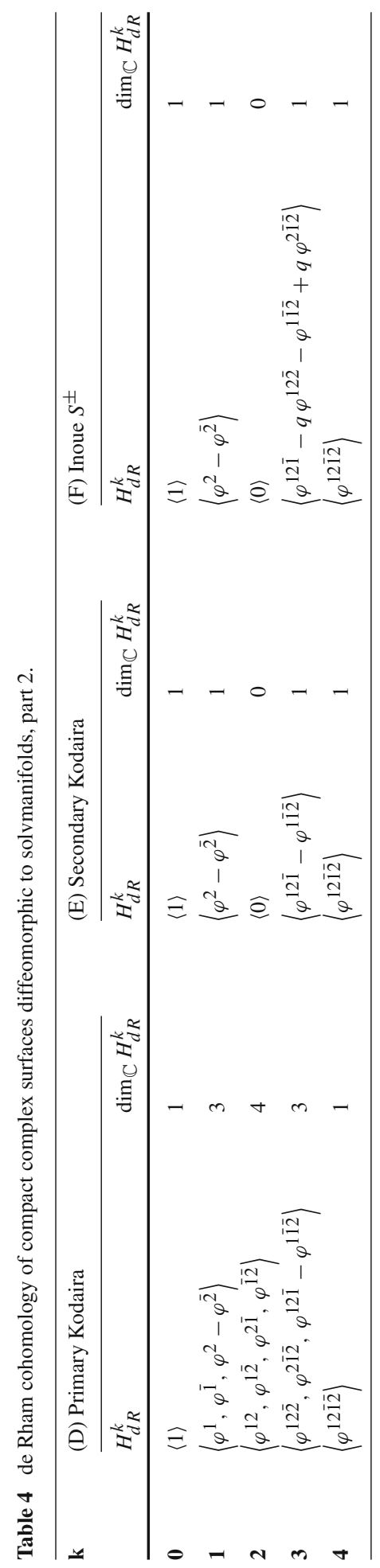




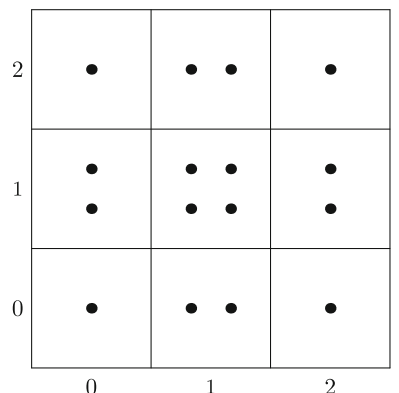

(A)

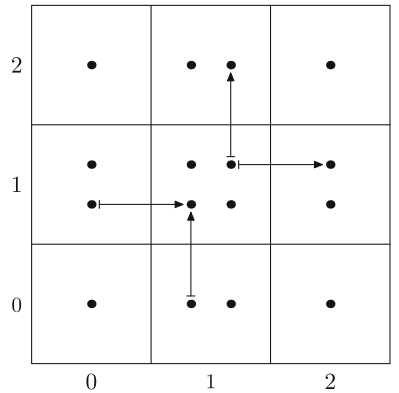

(D)

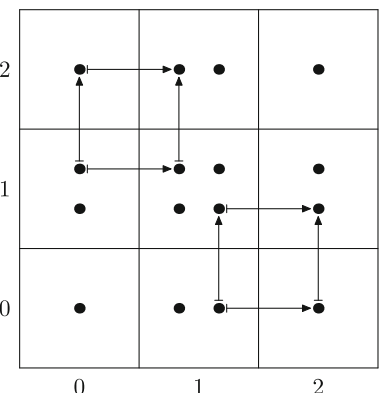

(B)

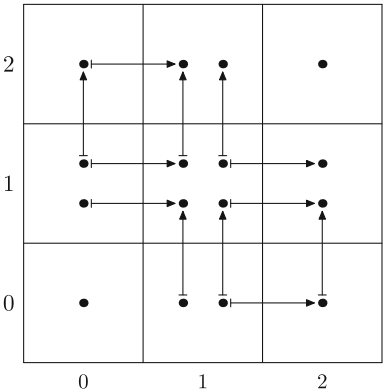

(E)

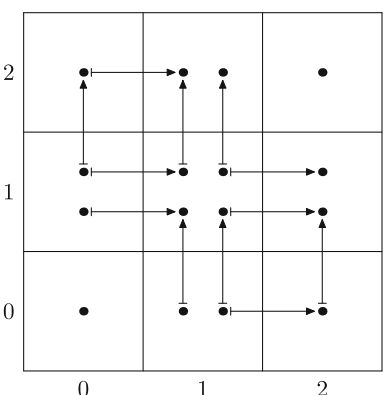

(C)

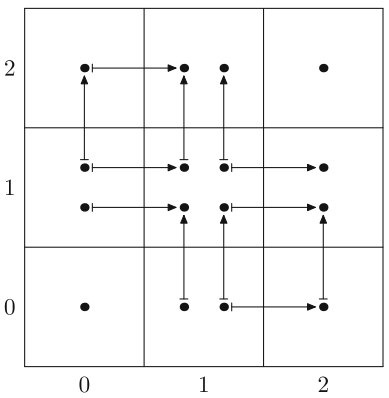

(F)

Fig. 1 Double-complexes of left-invariant forms over 4-dimensional solvmanifolds. a torus. b Hyperelliptic surface. c Inoue surface $\mathcal{S}_{M}$. d Primary Kodaira surface. e Secondary Kodaira surface. f Inoue surface $\mathcal{S}^{ \pm}$

Theorem 5.2 Let $X$ be a compact complex surface diffeomorphic to a solvmanifold. Then, the natural map $H_{B C}^{2,1}(X) \rightarrow H_{\bar{\partial}}^{2,1}(X)$ induced by the identity is an isomorphism, and the natural map $H_{B C}^{2,1}(X) \rightarrow H_{d R}^{3}(X ; \mathbb{C})$ induced by the identity is injective.

Proof By the general result in Theorem 1.1, the natural map $H_{B C}^{2,1}(X) \rightarrow H_{\bar{\partial}}^{2,1}(X)$ is injective. In fact, it is an isomorphism as follows from the computations summarized in Tables 1 and 2. As for the injectivity of the natural map $H_{B C}^{2,1}(X) \rightarrow H_{d R}^{3}(X ; \mathbb{C})$, it is a straightforward computation from Tables 1, 2, 3 and 4.

As an example, we offer an explicit calculation of the injectivity of the map $H_{B C}^{2,1}(X) \rightarrow$ $H_{d R}^{3}(X ; \mathbb{C})$ for the Inoue surfaces of type 0 , see [13], see also [22]. We will change a little bit the notation. Recall the construction of Inoue surfaces: let $M \in \operatorname{SL}(3 ; \mathbb{Z})$ be a unimodular matrix having a real eigenvalue $\lambda>1$ and two complex eigenvalues $\mu \neq \bar{\mu}$. Take a real eigenvector $\left(\alpha_{1}, \alpha_{2}, \alpha_{3}\right)$ and an eigenvector $\left(\beta_{1}, \beta_{2}, \beta_{3}\right)$ of $M$. Let $\mathbb{H}=\{z \in \mathbb{C} \mid$ Im $z>0\}$; on the product $\mathbb{H} \times \mathbb{C}$ consider the following transformations defined as

$$
\begin{aligned}
& f_{0}(z, w):=(\lambda z, \mu w) \\
& f_{j}(z, w):=\left(z+\alpha_{j}, w+\beta_{j}\right) \text { for } \quad j \in\{1,2,3\} .
\end{aligned}
$$

Denote by $\Gamma_{M}$ the group generated by $f_{0}, \ldots, f_{3}$; then, $\Gamma_{M}$ acts in a properly discontinuous way and without fixed points on $\mathbb{H} \times \mathbb{C}$, and $\mathcal{S}_{M}:=\mathbb{H} \times \mathbb{C} / \Gamma_{M}$ is an Inoue surface of type 0 , as in case (C) in [11]. Denoting by $z=x+i y$ and $w=u+i v$, consider the following differential forms on $\mathbb{H} \times \mathbb{C}$ : 


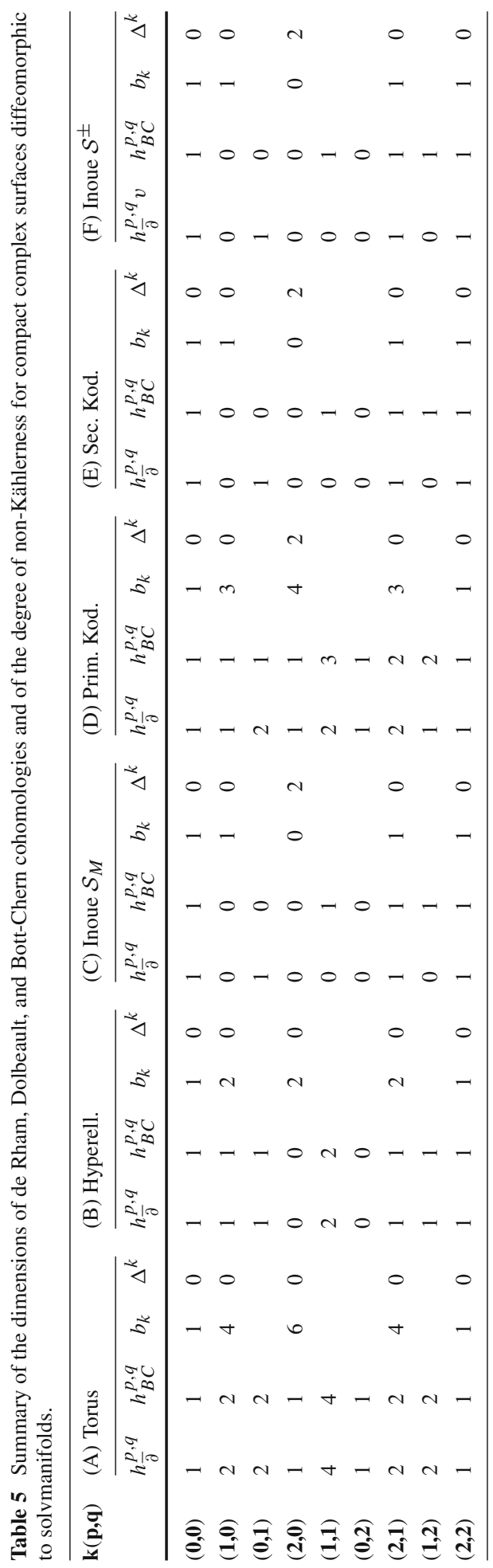




$$
e^{1}:=\frac{1}{y} \mathrm{~d} x, \quad e^{2}:=\frac{1}{y} \mathrm{~d} y, \quad e^{3}:=\sqrt{y} \mathrm{~d} u, \quad e^{4}:=\sqrt{y} \mathrm{~d} v .
$$

(Note that $e^{1}$ and $e^{2}$, and $e^{3} \wedge e^{4}$ are $\Gamma_{M}$-invariant, and consequently they induce global differential forms on $\mathcal{S}_{M}$.) We obtain

$$
\mathrm{d} e^{1}=e^{1} \wedge e^{2}, \quad \mathrm{~d} e^{2}=0, \quad \mathrm{~d} e^{3}=\frac{1}{2} e^{2} \wedge e^{3}, \quad \mathrm{~d} e^{4}=\frac{1}{2} e^{2} \wedge e^{4} .
$$

Consider the natural complex structure on $\mathcal{S}_{M}$ induced by $\mathbb{H} \times \mathbb{C}$. Locally, we have

$$
J e^{1}=-e^{2} \text { and } J e^{2}=e^{1} \text { and } J e^{3}=-e^{4} \text { and } J e^{4}=e^{3} .
$$

Considering the $\Gamma_{M}$-invariant $(2,1)$-Bott-Chern cohomology of $\mathcal{S}_{M}$, we obtain that

$$
H_{B C}^{2,1}\left(\mathcal{S}_{M}\right)=\mathbb{C}\left\langle\left[e^{1} \wedge e^{3} \wedge e^{4}+i e^{2} \wedge e^{3} \wedge e^{4}\right]\right\rangle .
$$

Clearly $\bar{\partial}\left(e^{1} \wedge e^{3} \wedge e^{4}+i e^{2} \wedge e^{3} \wedge e^{4}\right)=0$ and $e^{1} \wedge e^{3} \wedge e^{4}+i e^{2} \wedge e^{3} \wedge e^{4}=e^{1} \wedge e^{3} \wedge e^{4}+$ $i \mathrm{~d}\left(e^{3} \wedge e^{4}\right)$, therefore, the de Rham cohomology class $\left[e^{1} \wedge e^{3} \wedge e^{4}+i e^{2} \wedge e^{3} \wedge e^{4}\right]=$ $\left[e^{1} \wedge e^{3} \wedge e^{4}\right] \in H_{d R}^{3}\left(\mathcal{S}_{M}\right)$ is nonzero.

Acknowledgments The first and third authors would like to thank the Aix-Marseille University for its warm hospitality. Many thanks are due to the referee for her/his suggestions that improved the presentation. The third author would like to dedicate this paper to the memory of his mother.

\section{References}

1. Angella, D.: The cohomologies of the Iwasawa manifold and of its small deformations. J. Geom. Anal. 23(3), 1355-1378 (2013)

2. Angella, D., Kasuya H.: Bott-Chern cohomology of solvmanifolds. arXiv:1212.5708v3 [math.DG]

3. Angella, D., Tomassini, A.: On the $\partial \bar{\partial}$-Lemma and Bott-Chern cohomology. Invent. Math. 192(1), 71-81 (2013)

4. Barth, W.P., Hulek, K., Peters, C.A.M., Van de Ven, A.: Compact Complex Surfaces, Second Edition, Ergebnisse der Mathematik und ihrer Grenzgebiete, vol. 3. Springer, Berlin (2004)

5. Buchdahl, N.: On compact Kähler surfaces. Ann. Inst. Fourier (Grenoble) 49(1), vii, xi, 287-302 (1999)

6. Calabi, E., Eckmann, B.: A class of compact, complex manifolds which are not algebraic. Ann. Math. 58(2), 494-500 (1953)

7. Console, S., Fino, A.: Dolbeault cohomology of compact nilmanifolds. Transform. Groups 6(2), 111-124 (2001)

8. Deligne, P., Griffiths, Ph, Morgan, J., Sullivan, D.P.: Real homotopy theory of Kähler manifolds. Invent. Math. 29(3), 245-274 (1975)

9. Dloussky, G.: On surfaces of class $V I I_{0}^{+}$with numerically anticanonical divisor. Am. J. Math. 128(3), 639-670 (2006)

10. Fu, J., Yau, S.-T.: A note on small deformations of balanced manifolds. C. R. Math. Acad. Sci. Paris 349(13-14), 793-796 (2011)

11. Hasegawa, K.: Complex and Kähler structures on compact solvmanifolds, conference on symplectic topology. J. Symplectic Geom. 3(4), 749-767 (2005)

12. Hirzebruch, F.: Topological methods in algebraic geometry, translated from the German and Appendix One by R. L. E. Schwarzenberger, With a preface to the third English edition by the author and Schwarzenberger, Appendix Two by A. Borel, Reprint of the (1978) edition. Classics in Mathematics. Springer, Berlin (1995)

13. Inoue, M.: On surfaces of class $V I I_{0}$. Invent. Math. 24(4), 269-310 (1974)

14. Kodaira, K.: On the structure of compact complex analytic surfaces. I. Am. J. Math. 86, 751-798 (1964)

15. Lamari, A.: Courants kählériens et surfaces compactes. Ann. Inst. Fourier (Grenoble) 49(1), vii, x, 263285 (1999)

16. Lübke, M., Teleman, A.: The Kobayashi-Hitchin Correspondence. World Scientific, River Edge (1995)

17. Miyaoka, Y.: Kähler metrics on elliptic surfaces. Proc. Jpn. Acad. 50(8), 533-536 (1974) 
18. Parton, M.: Explicit parallelizations on products of spheres and Calabi-Eckmann structures. Rend. Istit. Mat. Univ. Trieste 35(1-2), 61-67 (2003)

19. Schweitzer, M.: Autour de la cohomologie de Bott-Chern. arXiv:0709.3528v1

20. Siu, Y.T.: Every K3 surface is Kähler. Invent. Math. 73(1), 139-150 (1983)

21. Teleman, A.: The pseudo-effective cone of a non-Kählerian surface and applications. Math. Ann. 335(4), 965-989 (2006)

22. Tricerri, F.: Some examples of locally conformal Kähler manifolds. Rend. Semin. Mat. Univ. Politec. Torino 40(1), 81-92 (1982) 